\title{
Is First Person Vision Challenging for Object Tracking?
}

\author{
Matteo Dunnhofer $\quad$ Antonino Furnari ${ }^{\star} \quad$ Giovanni Maria Farinella $^{\star} \quad$ Christian
$\begin{gathered}\text { Machine Learning and Perception Lab, University of Udine, Udine, Italy } \\ { }^{\star} \text { Image Processing Laboratory, University of Catania, Catania, Italy }\end{gathered}$
}

\section{Abstract}

Understanding human-object interactions is fundamental in First Person Vision (FPV). Tracking algorithms which follow the objects manipulated by the camera wearer can provide useful cues to effectively model such interactions. Despite a few previous attempts to exploit trackers in FPV applications, a methodical analysis of the performance of state-of-the-art visual trackers in this domain is still missing. In this short paper, we provide a recap of the first systematic study of object tracking in FPV. Our work extensively analyses the performance of recent and baseline FPV trackers with respect to different aspects. This is achieved through TREK-150, a novel benchmark dataset composed of 150 densely annotated video sequences. The results suggest that more research efforts should be devoted to this problem so that tracking could benefit FPV tasks.

\section{Introduction}

Understanding the interactions between a camera wearer and the surrounding objects is a fundamental problem in First Person Vision (FPV) [5, 10, 6]. To model such interactions, the continuous knowledge of where an object of interest is located inside the video frame is advantageous. To obtain such an information, most FPV solutions rely on object detection models that evaluate video frames independently. This paradigm has the drawback of ignoring all the temporal information coming from the object appearance and motion contained in consecutive video frames and generally requires a higher computational cost due to the repeated detection process on every frame. In contrast, visual object tracking aims to exploit past information about a target to infer its position and shape in the next frames of a video [18]. The benefits of tracking in FPV have been explored by a few previous works to predict future active objects [9], analyze social interactions [1], improve the performance of hand detection for rehabilitation purposes [21], locate hands and

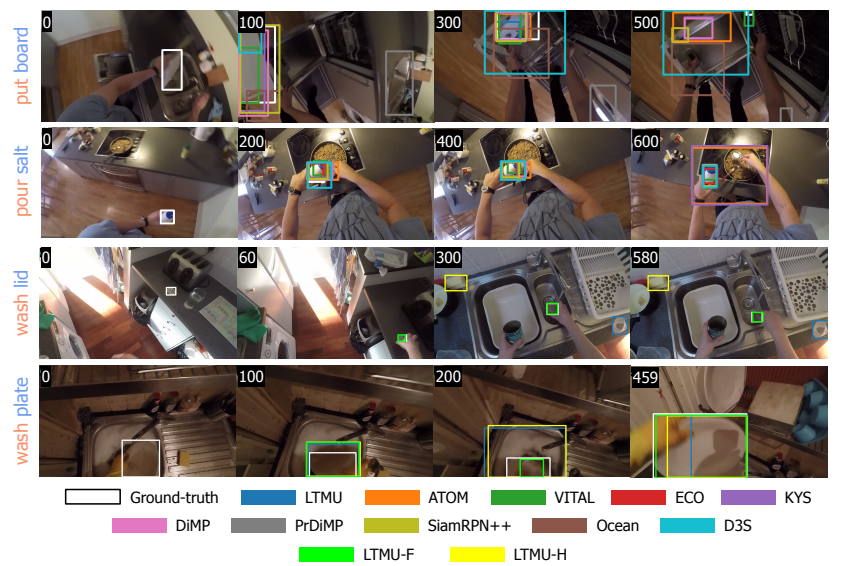

Figure 1: Qualitative results of some of the studied trackers on the proposed TREK-150 dataset. The first 2 rows of images show the qualitative performance of 10 of the selected generic-object trackers, while the last 2 rows show the results of the baseline FPV trackers LTMU-F and LTMU-H in comparison with LTMU. For each sequence, the action performed by the camera wearer is also reported (verb in orange, noun in blue). For a better visualization, a video can be found at https://youtu.be/oX1nICHgEJM.

capture their movements for action recognition [12] and human-object interaction forecasting [17]. Such works have proposed customized approaches to track specific targets like people, people faces, or hands from the FPV perspective. A solution specifically designed to track arbitrary objects in egocentric videos is still missing. This is due to the FPV challenges such as camera motion, persistent occlusion, significant scale and state changes, as well as motion blur, which prevent state-of-the-art visual trackers [11, 2, 3, 8] to track well generic objects. To support such a claim, we analyzed in-depth the problem of visual object tracking in the FPV domain. Particularly, we investigated the accuracy and speed performance of both non-FPV and FPV visual trackers, using standard and new performance evaluation strategies. We followed the visual tracking community's standard practice of building an accurate dataset for evaluation [22, 15] and proposed a 
novel visual tracking benchmark, TREK-150 (TRackingEpic-Kitchens-150) [7], which is obtained from the large and challenging FPV dataset EPIC-KITCHENS-55 (EK55) [5]. TREK-150 provides 150 video sequences densely annotated with the bounding boxes of a target object the camera wearer interacts with. Additionally, sequences have been labelled with attributes that identify the visual changes the object and scene are undergoing, the class of the target object and the action the person is performing. The results of the study showed that FPV offers challenging tracking scenarios for the most recent and accurate trackers and even for FPV trackers. Considering the potential impact of tracking on FPV, we suggest that more research efforts should be devoted to the considered task, for which we believe the proposed TREK-150 benchmark will be a key research tool. Please refer to [7] for the extended version of this abstract, including more details and results. Resources regarding TREK-150 are available at https://machinelearning.uniud.it/datasets/trek150/.

\section{The TREK-150 Benchmark Dataset}

The proposed TREK-150 dataset [7] is composed of 150 video sequences sampled from the EK-55 dataset [5]. In each video, a single target object is labeled with an axisaligned bounding box which encloses the visible parts of the object. Dense annotations were generated because it has been shown that they are required for visual tracking benchmarking [15]. To be compliant with other tracking challenges, every sequence is additionally labeled with one or more of 17 attributes describing the visual variability of the target in the sequence. They include 13 standard tracking attributes, plus 4 additional ones (High Resolution, Head Motion, 1-Hand Interaction, 2-Hands Interaction) which are introduced to characterize FPV sequences. In addition, 20 action verbs and 34 noun attributes are used to indicate the action performed by the camera wearer and the class of the target. Despite being a subset of EK-55, TREK-150 reflects its long-tail distribution of labels. Some qualitative examples of the video sequences are shown in Figure 1 .

\section{Trackers}

We considered 31 trackers to represent different stateof-the-art approaches to generic object tracking, for instance with respect to the matching strategy, type of image representations, learning strategy, etc. Specifically, in the analysis we have included short-term trackers based on correlation-filters with both hand-crafted features and deep features. We also considered deep siamese networks, tracking-by-detection methods, as well as trackers based on target segmentation representations, meta-learning, and fusion strategies. Long-term trackers have been also taken into account in the study.
In addition to the generic object trackers, we developed 2 baseline FPV trackers that combine the LTMU tracker [4] with (i) the EK-55 trained Faster-R-CNN [5] and (ii) the Faster-R-CNN-based hand-object detector [20], referred as LTMU-F and LTMU-H respectively. These baseline trackers exploit the respective detectors as object re-detection modules according to the LTMU scheme [4]. In short, the re-detection happens when a verification module notices that the tracker is not following the correct target. In such a case, the module triggers the execution of the respective FPV detector which proposes candidate locations of the target object. Each of the candidates is evaluated by the verification module, and the location with highest confidence is used to re-initialize the tracker.

\section{Evaluation}

We used the one-pass evaluation (OPE) protocol [22] that consists in initializing a tracker with the target's ground-truth bounding box in the first frame and let the tracker run on every other frame until the end of a video. To obtain a more robust evaluation, we employed the multistart evaluation (MSE) protocol [13] which defines different points of initialization along a video. A tracker is initialized with the ground-truth in each point and let run either forward or backward in time (depending on the longest subsequence yielded by the initialization point) until the end of the sub-sequence. Since many FPV tasks require real-time computation, we evaluated the ability of trackers to provide their object localization in such a setting by following the RTE protocol [14, 16]. It runs an algorithm considering its running time and skips all the frames, that occur regularly according to the frame rate, which appeared in between the algorithm's execution start and end times.

To compare the tracker's predicted bounding boxes with the temporally aligned ground-truth bounding boxes we used different measures: the success plot [22] which shows the percentage of predicted bounding boxes whose intersection-over-union with the ground-truth is larger than a threshold varied from 0 to 1 (Figure 2 (a)); the normalized precision plot [19] that reports, for a variety of distance thresholds, the percentage of bounding boxes whose center points are within a given normalized distance (in pixels) from the ground-truth (Figure 2 (b)); a novel plot which we refer to as generalized success robustness plot (Figure 2 (c)) which measures the normalized extent of a tracking sequence before a failure. As summary measures, we report the success score (SS) [22], normalized precision score (NPS) [19], and generalized success robustness (GSR), which are computed as the Area Under the Curve (AUC) of the success plot, the normalized precision plot, and generalized success robustness plot respectively. 


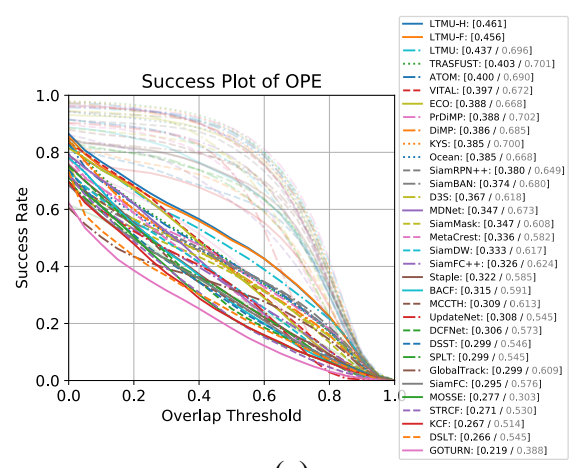

(a)

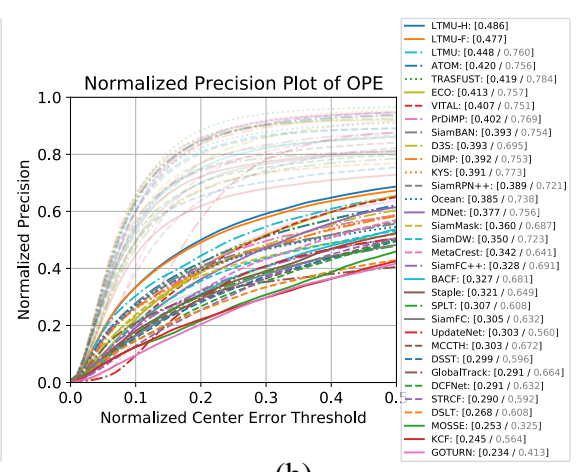

(b)

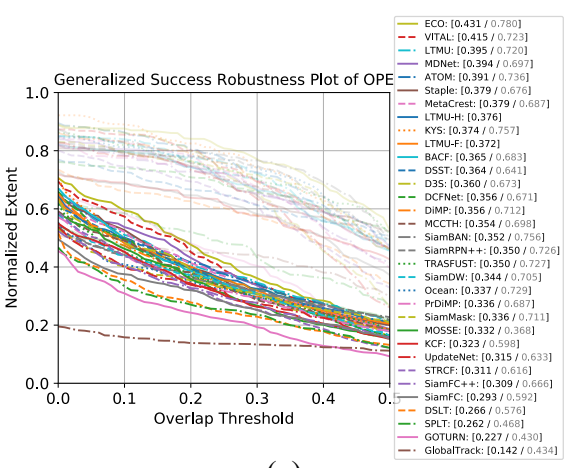

(c)

Figure 2: Performance of the selected trackers on the proposed TREK-150 benchmark under the OPE protocol. The curves in solid colors report the performance of the 33 benchmarked trackers on TREK-150, whereas the curves overlaid in semitransparent colors outline the performance obtained by the same trackers on the standard OTB-100 [22] dataset. In brackets, next to the trackers' names, we report the SS, NPS and GR values achieved on TREK-150 (in black) and on OTB-100 [22] (in gray). As can be noted, all the trackers exhibit a significant performance drop when tested on our challenging FPV benchmark. LTMU-H and LTMU-F achieve marginally better performance, while we expect significant boosts to be achievable with a careful design of FPV trackers.

Table 1: Performance achieved by 17 of the benchmarked trackers on TREK-150 using the RTE protocol.

\begin{tabular}{|c|c|c|c|c|c|c|c|c|c|c|c|c|c|c|c|c|c|}
\hline Metric & Ocean & SiamBAN & SiamRPN++ & DiMP & KYS & ATOM & LTMU & D3S & ECO & GlobalTrack & Staple & MOSSE & LTMU-H & MetaCrest & LTMU-F & VITAL & KCF \\
\hline FPS & 21 & 24 & 23 & 16 & 12 & 15 & 8 & 16 & 15 & 8 & 13 & 26 & 4 & 8 & 4 & 4 & 6 \\
\hline SS & 0.365 & 0.360 & 0.362 & 0.336 & 0.327 & 0.319 & 0.284 & 0.276 & 0.252 & 0.253 & 0.249 & 0.227 & 0.213 & 0.207 & 0.205 & 0.204 & 0.186 \\
\hline NPS & 0.358 & 0.366 & 0.356 & 0.331 & 0.317 & 0.312 & 0.257 & 0.263 & 0.231 & 0.227 & 0.236 & 0.190 & 0.174 & 0.175 & 0.161 & 0.165 & 0.157 \\
\hline GSR & 0.294 & 0.313 & 0.293 & 0.224 & 0.237 & 0.179 & 0.169 & 0.182 & 0.173 & 0.139 & 0.169 & 0.141 & 0.161 & 0.165 & 0.162 & 0.158 & 0.177 \\
\hline
\end{tabular}

\section{Results}

General Remarks. Figure 2 reports the performance of the selected trackers on TREK-150 using the OPE protocol, as well their performance on the popular OTB-100 [22] benchmark for reference (semi-transparent curves - gray numbers in brackets). The results are decreased across all measures when considering the challenging FPV scenario of TREK-150, demonstrating that the particular characteristics of FPV introduce challenging scenarios for visual trackers. Some qualitative examples of the trackers' performance are shown in Figure 1 Trackers based on deep learning perform better in SS and NPS than those based on handcrafted features. Among the formers, those that leverage online adaptation mechanisms are more accurate than the ones based on single-shot instances. The generalized success robustness plot in Figure 2(c) reports a different ranking of the trackers, showing that more spatially accurate trackers are not always able to maintain longer reference to targets. The proposed FPV trackers LTMU-H and LTMU-F are better in SS and NPS, while they lose some performance in GSR. Such outcome shows that adapting a state-of-the-art method to FPV allows to marginally improve results. We expect significant performance improvements to be achievable by a tracker accurately designed to tackle the FPV challenges.

Table 1 reports the FPS performance of the trackers and the SS, NPS, and GSR scores achieved under the RTE protocol. It can be noted the performance of all trackers is de- creased when considering their running time in relation to the frame rate. These results suggest that efforts should be made to make trackers accurate in real-time applications.

Conditions That Influence The Trackers. Figure 3 reports the SS, NPS, and GSR scores of trackers, computed with the MSE protocol, with respect to the attributes used to characterize the visual variability of the sequences. Full occlusion (FOC), out of view (OUT) and the small size of targets (LR) are the most difficult situations. The fast motion of targets (FM) and the presence of similar objects (SOB) are also critical factors that cause drops in performance. Trackers are less vulnerable to rotations (ROT) and to the illumination variation (IV). Tracking objects held with two hands $(2 \mathrm{H})$ is more difficult than tracking objects held with a single hand $(1 \mathrm{H})$, while trackers are quite robust to head motion (HM) and objects appearing in larger size (HR). Considering the performance with respect to actions, it results that those mainly causing a spatial displacement of the target (e.g. "move", "store", "check") have less impact. Actions that change the state, shape, or aspect ratio of an object (e.g. "remove", "squeeze", "cut", "attach") generate harder tracking scenarios. Also the sequences including the "wash" verb lead to poor performance. With respect to the associated noun labels, we have that rigid, regular-sized objects such as "pan", "kettle", "bowl", "plate", and "bottle" are among the ones associated with high average scores. 

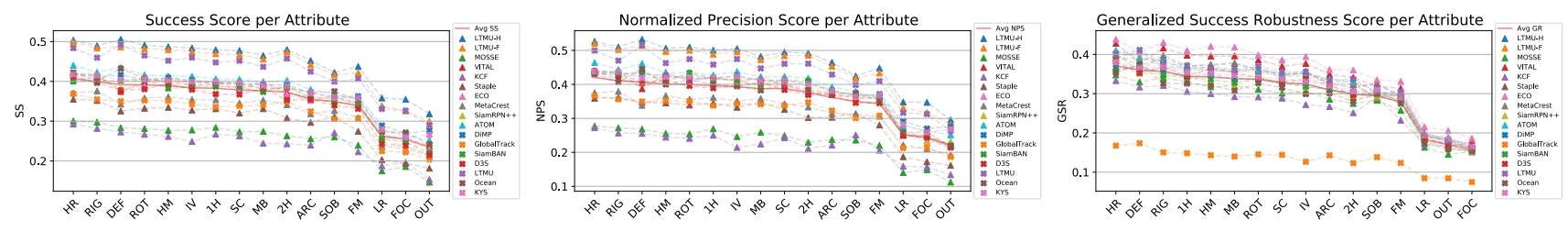

Figure 3: SS, NPS, and GSR of 17 of the benchmarked trackers on the sequence attributes of proposed TREK-150 benchmark under the MSE protocol. The red plain line highlights the average performance.

Table 2: Accuracy results on TREK-150 of a video-based hand-object detection solution which considers each of the considered trackers as localization method for the object involved in the interaction. As a baseline, we employ the object detection capabilities of the hand-object interaction solution Hands-in-contact [20].

\begin{tabular}{c|c|cccccccccccccccccc}
\hline Hands-in-contact & 20 & LTMU-H & LTMU-F & ATOM & LTMU & Ocean & SiamBAN & SiamRPN++ & MetaCrest & D3S & DiMP & KYS & VITAL & GlobalTrack & MOSSE & ECO & Staple & KCF \\
\hline 0.354 & 0.368 & 0.367 & 0.361 & 0.354 & 0.340 & 0.340 & 0.311 & 0.293 & 0.292 & 0.292 & 0.279 & 0.253 & 0.251 & 0.231 & 0.230 & 0.197 & 0.177 \\
\hline
\end{tabular}

Other rigid objects such as "knife", "spoon", "fork" and "can" are harder to track instead, probably due to their particularly thin shape and the light reflectance they are easily subject to. Deformable objects such as "sponge", "onion", "cloth" and "rubbish" are also difficult to track.

Impact of Trackers in FPV. Despite we demonstrated that FPV is challenging for current trackers, we assessed whether these already offer an advantage in the FPV domain to obtain information about the objects' locations and movements in the scene. To this aim, we evaluated the performance of a Faster R-CNN instance trained on EK55 [5] when used as a naive tracking baseline. Such a solution achieved an SS, NPS, and GSR of 0.323, 0.369, 0.044 , by running at 1 FPS. Comparing these results with the ones presented in Figure 2, we clearly notice that trackers, if properly initialized by a detection module, can deliver faster, more accurate and much more temporally long object localization than detectors. We additionally evaluated the accuracy of a video-based hand-object interaction detection solution [20] whose object localisation is given by a tracker rather than a detector. The tracker is initialized with the object detector's predicted bounding box at the first detection of the hand-object interaction, and let run until its end. By this setting, we created a ranking of the trackers which is presented in Table 2 The results demonstrate that stronger trackers can improve the accuracy and efficiency of current detection-based methodologies [20]. Interestingly, the trackers' ranking differs from what shown in Figure 2 , suggesting that trackers can manifest other capabilities when deployed into application scenarios.

Given these preliminary results, we hence expect that trackers will likely gain more importance in FPV as new methodologies explicitly considering the first person point of view are investigated.

\section{References}

[1] Maedeh Aghaei, Mariella Dimiccoli, and Petia Radeva. With whom do i interact? Detecting social interactions in egocentric photo-streams. In Proceedings - International Conference on Pattern Recognition, volume 0, pages 2959-2964. Institute of Electrical and Electronics Engineers Inc., jan 2016.

[2] Luca Bertinetto, Jack Valmadre, João F. Henriques, Andrea Vedaldi, and Philip H.S. Torr. Fullyconvolutional siamese networks for object tracking. European Conference on Computer Vision, 9914 LNCS:850-865, 2016.

[3] Goutam Bhat, Martin Danelljan, Luc Van Gool, and Radu Timofte. Learning Discriminative Model Predic tion for Tracking. In Proceedings of the IEEE/CVF International Conference on Computer Vision, 2019.

[4] Kenan Dai, Yunhua Zhang, Dong Wang, Jianhua Li, Huchuan Lu, and Xiaoyun Yang. High-Performance Recognition, pages 6297-6306. Institute of Electrical and Electronics Engineers (IEEE), aug 2020.

[5] D. Damen, H. Doughty, G. M. Farinella, S. Fidler, A. Furnari, E. Kazakos, D. Moltisanti, J. Munro, T. Perrett, W. Price, and M. Wray. Scaling egocentric vision: The epic-kitchens dataset. In European Conference on Computer Vision (ECCV), 2018.

[6] Dima Damen, Teesid Leelasawassuk, and Walterio Mayol-Cuevas. You-do, i-learn: Egocentric unsupervised discovery of objects and their modes of interaction towards video-based guidance. Computer Vision and Image Understanding, 149:98-112, 2016.

[7] Matteo Dunnhofer, Antonino Furnari, Giovanni Maria Farinella, and Christian Micheloni. Is first person vision challenging for object tracking? In Proceedings of the IEEE/CVF International Conference on Computer Vision (ICCV) Workshops, Oct 2021.

[8] Matteo Dunnhofer, Niki Martinel, and Christian Micheloni. Tracking-by-Trackers with a Distilled and Reinforced Model. In Asian Conference on Computer Vision, 2020.

[9] Antonino Furnari, Sebastiano Battiato, Kristen Grauman, and Giovanni Maria Farinella. Next-active-object prediction from egocentric videos. Journal of Visual Communication and Image Representation, 49:401411, nov 2017.

[10] Antonino Furnari and Giovanni Farinella. Rolling-Unrolling LSTMs for Action Anticipation from FirstPerson Video. IEEE Transactions on Pattern Analysis and Machine Intelligence, pages 1-1, may 2020.

[11] Joao F. Henriques, Rui Caseiro, Pedro Martins, and Jorge Batista. High-speed tracking with kernelized correlation filters. IEEE Transactions on Pattern Analysis and Machine Intelligence, 37(3):583-596, 2015.

[12] Georgios Kapidis, Ronald Poppe, Elsbeth Van Dam, Lucas Noldus, and Remco Veltkamp. Egocentric hand track and object-based human action recognition. In 2019 IEEE SmartWorld, Ubiq uitous Intelligence \& Computing, Advanced \& Trusted Computing, Scalable Computing \& Commnications, Cloud \& Big Data Computing, Internet of People and Smart City Innovation (SmartWorld/SCALCOM/UIC/ATC/CBDCOM/IOP/SCI), pages 922-929. IEEE, 2019.

[13] Matej Kristan, Aleš Leonardis, Jiř́ Matas, Michael Felsberg, Roman Pflugfelder, Joni-Kristian Kämäräinen, Martin Danelljan, Luka Čehovin Zajc, Alan Lukežičc, Ondrej Drbohlav, Linbo He, Yushan Zhang, Song Yan, Jinyu Yang, Gustavo Fernández, et al. The eighth visual object tracking vot2020 chalpages 547-601, Cham, 2020. Springer International Publishing.
parts

[14] Matej Kristan, Ales Leonardis, Jiri Matas, Michael Felsberg, Roman Pflugfelder, Luka Cehovin Zajc, Tomas Vojir, Gustav Hager, Alan Lukezic, Abdelrahman Eldesokey, Gustavo Fernandez, et al. The Visual Object Tracking VOT2017 Challenge Results. In Proceedings of the IEEE International Conference on Computer Vision Workshops, pages 1949-1972. IEEE, oct 2017.

[15] Matej Kristan, Jiř̌́ Matas, Aleš Leonardis, Michael Felsberg, Roman Pflugfelder, Joni-Kristian Kämäräinen, LukaCehovinLuka`LukaCehovin Zajc, Ondrej Drbohlav, Alan Lukežič, Amanda Berg, Abdelrahman Eldesokey, Jani Käpylä, Gustavo Fernández, et al. The Seventh Visual Object Tracking VOT2019 Challenge Results. In Proceedings of the IEEE/CVF International Conference on Computer Vision Workshops, 2019.

[16] Mengtian Li, Yu-Xiong Wang, and Deva Ramanan. Towards streaming perception. In Andrea Vedaldi, Horst Bischof, Thomas Brox, and Jan-Michael Frahm, editors, Computer Vision - ECCV 2020, pages 473-488, Cham, 2020. Springer International Publishing.

[17] Miao Liu, Siyu Tang, Yin Li, and James Rehg. Forecasting human object interaction: Joint prediction of motor attention and actions in first person video. In Proceedings of the European Conference on Computer Vision (ECCV), volume 2, 2020.

[18] Dr Emilio Maggio and Dr Andrea Cavallaro. Video Tracking: Theory and Practice. Wiley Publishing, 1st edition, 2011.

[19] Matthias Müller, Adel Bibi, Silvio Giancola, Salman Alsubaihi, and Bernard Ghanem. TrackingNet: A Large-Scale Dataset and Benchmark for Object Tracking in the Wild. In European Conference on Computer Vision, volume 11205 LNCS, pages 310-327. Springer Verlag, mar 2018.

[20] Dandan Shan, Jiaqi Geng, Michelle Shu, and David F. Fouhey. Understanding human hands in contact at internet scale. In Proceedings of the IEEE/CVF Conference on Computer Vision and Pattern Recognition (CVPR), June 2020.

[21] Ryan J. Visee, Jirapat Likitlersuang, and Jose Zariffa. An Effective and Efficient Method for Detecting Hands in Egocentric Videos for Rehabilitation Applications. IEEE Transactions on Neural Systems and Rehabilitation Engineering, 28(3):748-755, mar 2020.

[22] Yi Wu, Jongwoo Lim, and Ming Hsuan Yang. Object tracking benchmark. IEEE Transactions on Pattern Analysis and Machine Intelligence, 37(9):1834-1848, sep 2015. 